\title{
Near Real Time Enhancement of Remote Sensing Imagery Based on a Network of Systolic Arrays
}

\author{
A. Castillo Atoche ${ }^{1,2}$, D. Torres Roman ${ }^{1}$, and Y. Shkvarko ${ }^{1}$ \\ ${ }^{1}$ Department of Electrical Engineering, CINVESTAV-IPN, 45015 Jalisco, Mexico \\ ${ }^{2}$ Department of Mechatronics, Autonomous University of Yucatan, 150 Yucatan, Mexico \\ \{acastillo, dtorres, shkvarko\} @gdl.cinvestav.mx
}

\begin{abstract}
In this paper, we propose a novel Hardware/Software (HW/SW) codesign approach for near real time implementation of high-resolution reconstruction of remote sensing (RS) imagery using an efficient network of systolic arrays (NSA). Such proposed NSA technique is based on a Field Programmable Gate Array (FPGA) and implements the image enhancement/reconstruction tasks of the intelligent descriptive experiment design regularization (DEDR) methodology in an efficient concurrent processing architecture that meets the (near) real time imaging systems requirements in spite of conventional computations. Finally, the results of the HW/SW co-design implementation in a Xilinx Virtex-4 XC4VSX35-10ff668 for the reconstruction of real world RS images are reported and discussed.
\end{abstract}

Keywords: Remote Sensing, Hardware/Software Co-Design, Network of Systolic Arrays.

\section{Introduction}

The newer techniques for image reconstruction/enhancement used in high resolution remote-sensing (RS) and radar imaging are computationally expensive [1],[2]. Therefore, these techniques are not suitable for a (near) real time implementation with current digital signal processors (DSP) or personal computers (PC). The descriptive experiment design regularization (DEDR) approach for RS image enhancement/reconstruction has been detailed in many works; here we refer to [3],[4] where such an approach is adapted to the remote sensing (RS) applications with the use of a synthetic aperture radar (SAR) considered in this paper.

The scientific challenge of this study is to solve the enhanced/reconstruction RS imaging problems in context of the (near) real time computing via employing the software/hardware co-design paradigm.

The innovative contribution that distinguishes our approach from the previous studies [4],[5],[6] is twofold. First, we address a new unified intelligent descriptive experiment design regularization (DEDR) methodology and the HW/SW Co-Design technique for (near) real time enhancement/reconstruction of the remote sensing (RS) imagery. Second, the network of Systolic Arrays (NSA) implements the DEDR methodology tasks in a computationally efficient fashion that meets the (near) real time imaging system requirements. 
Finally, we report some simulation results and discuss the implementation performance issues related to (near) real time enhancement of the large-scale real-world RS/SAR imagery indicative of the significantly increased performance efficiency gained with the developed HW/SW co-design.

\section{Background}

In this section, we present a brief summary of the Descriptive Experiment Design Regularization Method (DEDR) previously defined in [3]. Let us consider the measurement data wavefield $u(\mathrm{y})=s(\mathrm{y})+n(\mathrm{y})$ modeled as a superposition of the echo signals $s$ and additive noise $n$ that assumed to be available for observations and recordings within the prescribed time-space observation domain $Y \ni \mathbf{y}$, where $\mathrm{y}=(t, \mathrm{p})^{\mathbf{T}}$ defines the time-space points in the observation domain $Y=T \times P$. The model of observation wavefield $u$ is specified by the linear stochastic equation of observation (EO) of operator form [3]: $u=S e+n ; e \in \mathrm{E} ; u, n \in \mathrm{U} ; S: \mathrm{E} \rightarrow \mathrm{U}$. Next, we take into account the stochastic operator-form of the observation equation (EO) vector formwhere

$$
\mathbf{u}=\widetilde{\mathbf{S e}}+\mathbf{n}=\mathbf{S e}+\Delta \mathbf{e}+\mathbf{n}
$$

where the matrix $\widetilde{\mathbf{S}}=\mathbf{S}+\Delta$, represents the nominal signal formation operator SFO and $\mathbf{e}, \mathbf{n}, \mathbf{u}$ represent the zero-mean vectors. These vectors are characterized by the correlation matrices: $\mathbf{R}_{\mathbf{e}}=\mathbf{D}=\mathbf{D}(\mathbf{b})=\operatorname{diag}\{\mathbf{b}\}$ (a diagonal matrix with vector $\mathbf{b}$ at its principal diagonal), $\mathbf{R}_{\mathbf{n}}$, and $\mathbf{R}_{\mathbf{u}}=\left\langle\widetilde{\mathbf{S}} \mathbf{R}_{\mathbf{e}} \widetilde{\mathbf{S}}^{+}\right\rangle_{p(\boldsymbol{\Delta})}+\mathbf{R}_{\mathbf{n}}$, respectively, where $\left.\langle\cdot\rangle_{p(} \boldsymbol{\Delta}\right)$ defines the averaging performed over the randomness of $\boldsymbol{\Delta}$ characterized by the $u n$ known probability density function $p(\boldsymbol{\Delta})$. Vector $\mathbf{b}$ is composed of the elements, $b_{k}=$ $\left.<e_{k} e_{k}{ }^{*}\right\rangle=\left\langle\left|e_{k}\right|^{2}>; k=1, \ldots, K\right.$, and is referred to as a $K$-D vector-form approximation of the SSP.

We refer to the estimate, $\hat{\mathbf{b}}$, as a discrete-form representation of the desired SSP i.e. the brightness image of the wavefield sources distributed over the pixel-formatted object scene remotely sensed with an employed array radar/SAR. Thus, one can seek to estimate $\hat{\mathbf{b}}=\left\{\mathbf{R}_{\mathbf{e}}\right\}_{\text {diag }}$ given the data correlation matrix $\mathbf{R}_{\mathbf{u}}$ pre-estimated by some means, e.g. via averaging the correlations over $J$ independent snapshots [3]

$$
\mathbf{R}_{\mathbf{u}}=\mathbf{Y}=\underset{j \in J}{\operatorname{aver}}\left\{\mathbf{u}_{(j)} \mathbf{u}_{(j)}^{+}\right\}=(1 / J) \sum_{j=1}^{J} \mathbf{u}_{(j)} \mathbf{u}_{(j)}^{+},
$$

and by determining the solution operator that we also refer to as the signal image formation operator ( $\mathrm{SO}) \mathbf{F}$ such that

$$
\hat{\mathbf{b}}=\left\{\hat{\mathbf{R}}_{\mathrm{e}}\right\}_{\text {diag }}=\left\{\mathbf{F Y} \mathbf{F}^{+}\right\}_{\text {diag }} .
$$

A family of the DEDR-related algorithms for estimating the SSP was derived by [3] as follows. 


\subsection{Robust Spatial Filtering Algorithm}

Consider the white zero-mean noise in observations and no preference to any prior model information, putting $\mathbf{A}=\mathbf{I}$. Let the regularization parameter be adjusted as the inverse of the signal-to-noise ratio (SNR), e.g. $\alpha=N_{0} / b_{0}$, where $b_{0}$ is the prior average gray level of the SSP, and the uncertainty factor $\beta$ is attributed to $\alpha$. In that case the SO F is recognized to be the Tikhonov's robust spatial filter:

$$
\mathbf{F}_{R S F}=\mathbf{F}^{(1)}=\left(\mathbf{S}^{+} \mathbf{S}+\left(N_{0} / b_{0}\right) \mathbf{I}\right)^{-1} \mathbf{S}^{+} .
$$

\subsection{Robust Adaptive Spatial Filtering Algorithm}

Consider the case of an arbitrary zero-mean noise with the composed correlation matrix $\mathbf{R}_{\Sigma}$, equal importance of two error measures, i.e. $\alpha=1$, and the solution dependent weight matrix $\mathbf{A}=\hat{\mathbf{D}}=\operatorname{diag}(\hat{\mathbf{b}})$. In this case, the SO becomes the robust adaptive (i.e. solution-dependent) spatial filter (RASF) operator:

$$
\mathbf{F}_{\text {RASF }}=\mathbf{F}^{(2)}=\left(\mathbf{S}^{+} \mathbf{R}_{\Sigma}^{-1} \mathbf{S}+\hat{\mathbf{D}}^{-1}\right)^{-1} \mathbf{S}^{+} \mathbf{R}_{\Sigma}^{-1} .
$$

Now, we are ready to proceed with the algorithms transformation into their locally recursive format representation [10], in which the data dependencies of the computations are exposed in a dependence graphs (DG) [11] to represent the parallel characteristics of the algorithms.

\section{Mapping Algorithms onto Systolic Arrays Structures}

An array processor consists of a number of processors elements (PE) and interconnection links among the PEs. The systolic design maps an N-dimensional dependence graph DG into a lower dimensional systolic array. In order to derive a regular SA architecture with a minimum possible number of nodes, we employ a linear projection approach for processor assignment, i.e., the nodes of the DG in a certain straight line are projected onto the corresponding PEs in the processor array represented by the corresponding assignment projection vector $\mathbf{d}$. Thus, we seek for a linear order reduction transformation $\mathbf{T}[10]$ where

$$
\mathbf{T}=\left[\begin{array}{c}
\boldsymbol{\Pi} \\
\boldsymbol{\Sigma}
\end{array}\right],
$$

where $\boldsymbol{\Pi}$ is a $(1 \times p)$-dimensional vector (composed of the first row of $\mathbf{T})$ which determine the time scheduling and the sub-matrix $\boldsymbol{\Sigma}$ of $(p-1) \times p$ dimension (composed of the rest rows of $\mathbf{T}$ ), determine the space processor [11]. Now, we proceed to construct the SAs structures. 


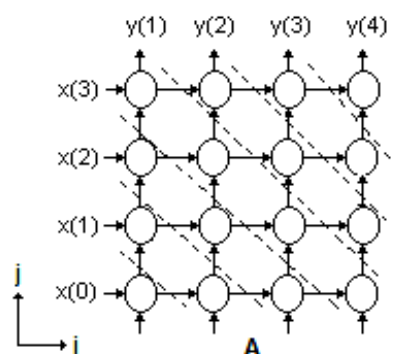

a)

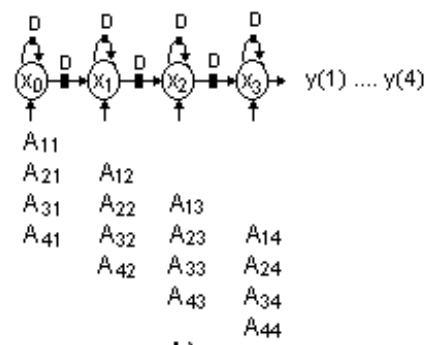

b)

Fig. 1. Matrix-vector systolic implementation: a) Standard DG; b) Systolic array

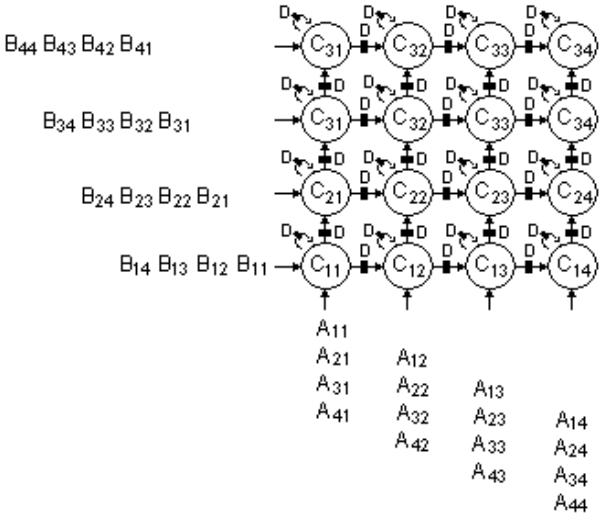

Fig. 2. Matrix-matrix systolic implementation

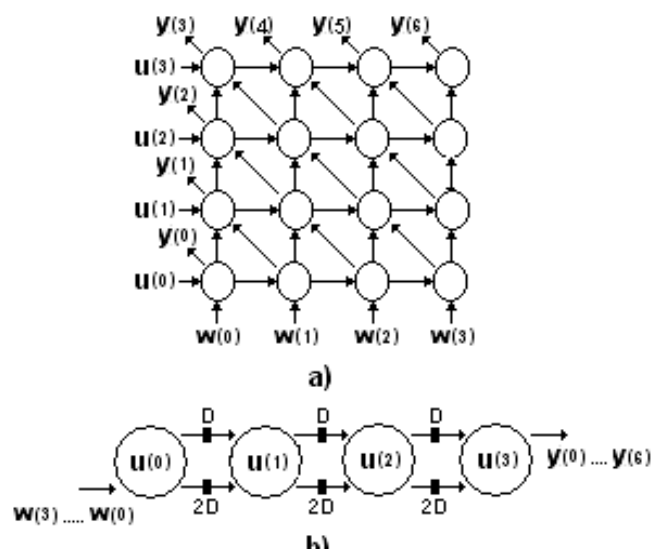

b)

Fig. 3. Convolution systolic implementation: a) Standard DG; b) Systolic array

\section{A. Matrix-Vector Systolic Implementation}

Let us consider a matrix $\mathbf{A}$ of size $n \times n$ and a vector $\mathbf{x}$ of size $n \times 1$, i.e. $\mathbf{y}=\mathbf{A x}$. The DG for a standard Matrix-Vector multiplication with a vector schedule of $\Pi=\left[\begin{array}{ll}1 & 1\end{array}\right]^{\mathrm{T}}$ is depicted in Figure 2(a). Next, we select a projection vector $\mathbf{d}=\left[\begin{array}{ll}1 & 0\end{array}\right]^{\mathrm{T}}$. The corresponding systolic array is obtained as we can see in Figure 2(b). The pipelining period for this systolic array is one. The number of PEs required by this structure is $n$. The computational time required by this systolic array is $2 n-1$ clock periods.

\section{B. Matrix-Matrix Systolic Implementation}

Let $\mathbf{A}$ be an $m \times n$ matrix and $\mathbf{B}$ be an $n \times k$ matrix. The product of the matrices is an $m \times k$ matrix $\mathbf{C}$, i.e. $\mathbf{C}=\mathbf{A B}$. The $\mathrm{DG}$ of a standard matrix-matrix multiplication 
algorithm corresponds to a 3-D space representation. In Figure 3, the systolic structure with the projection direction of $\mathbf{d}=\left[\begin{array}{lll}0 & 0 & 1\end{array}\right]^{\mathrm{T}}$ is obtained. This architecture requires an array of $m k$ PEs and $n+m+k-1$ clock periods.

\section{Convolution Systolic Implementation}

Given the vectors $\mathbf{u}$ of size $n \times 1$ and $\mathbf{w}$ of size $m \times 1$, the DG of the convolution algorithm with a systolic schedule vector $\boldsymbol{\Pi}=\left[\begin{array}{ll}1 & 2\end{array}\right]^{\mathrm{T}}$ is presented in Figure 4(a). The resulting systolic array considering the projection vector $\mathbf{d}=\left[\begin{array}{ll}1 & 0\end{array}\right]^{\mathrm{T}}$ is presented in Figure 4(b). This architecture requires an array of $n$ PEs and $n+2 m-2$ clock periods.

\section{Hardware/Software Co-design with a Network of Systolic Arrays}

The HW/SW co-design is a hybrid method aimed at increasing the flexibility of the implementation and improving the overall design process. The all-software execution of the RS image formation and reconstruction operations may be intensively time consuming, even using modern high-speed personal computers (PC) or any existing digital signal (DSP). In this section, a concise description of a $\mathrm{HW} / \mathrm{SW}$ co-design approach is presented, and its flexibility in performing an efficient HW implementation of the SW processing tasks with the NSA design is demonstrated. Figure 4 illustrates the pursued HW/SW co-design paradigm. The block units of Figure 4 are to be designed to speed up the digital signal processing operations of the DR algorithm previously developed to meet the real time imaging system requirements.

In this study, we select the Microblaze embedded processor (for the restricted platform) and the On Chip Peripheral Bus (OPB) [7], [8] for transferring the data from/to the embedded processor to/from the NSA as it is illustrated in Figure 4. Such the OPB is a fully synchronous bus that connects other separate 32-bit data buses. Such system architecture (based on the FPGA XC4VSX35-10ff668 with the embedded processor and the OPB buses) restricts the corresponding processing frequency to $100 \mathrm{MHz}$.

The crucial issue of this design is the proposed NSA design. With the NSA multiple data transfer from the embedded processor data memory to the SAs are avoided. Such NSA design guarantee the drastically reduction of the overall computation time. Finally, the interface unit must employ all the required operational and control functions: loading and storing the data to/from the embedded processor and data/control parallel transfer through the processor elements in a specific spatial/temporal manner. The system control is performed to guarantee the proper synchronization of the data in the proposed interface and to habilitate the corresponding system control of the SA. 


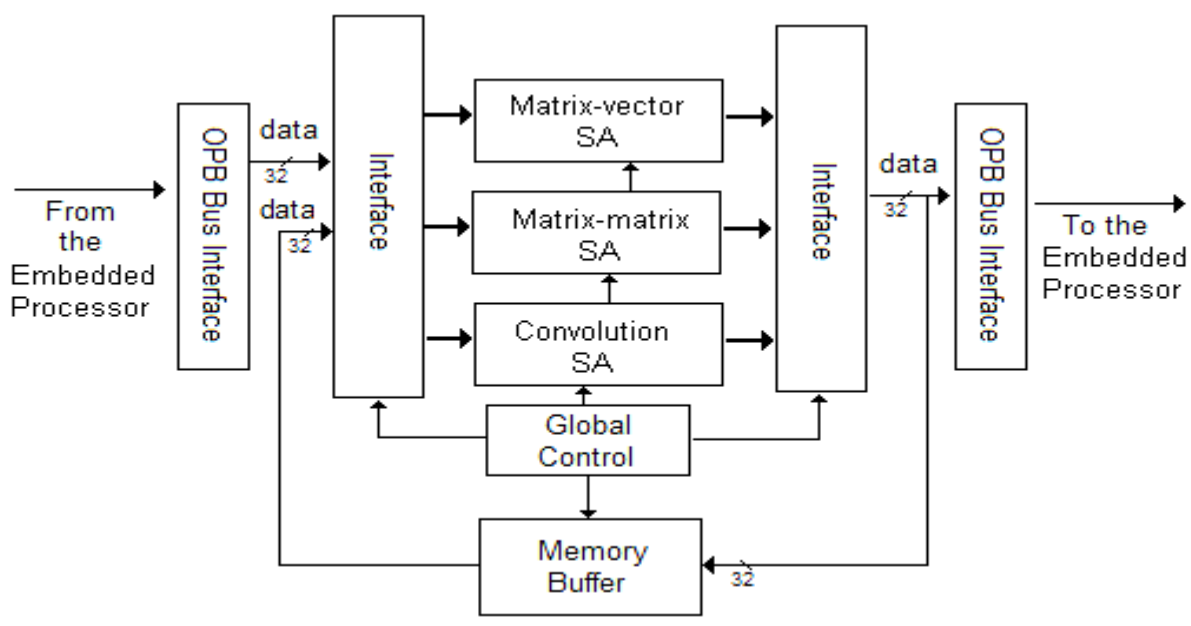

Fig. 4. HW/SW Co-design with the proposed NSA design

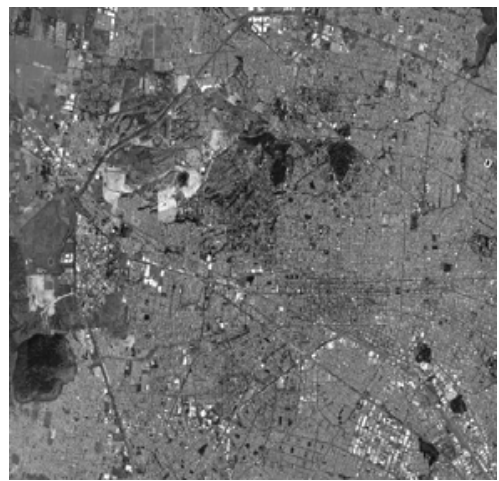

a)

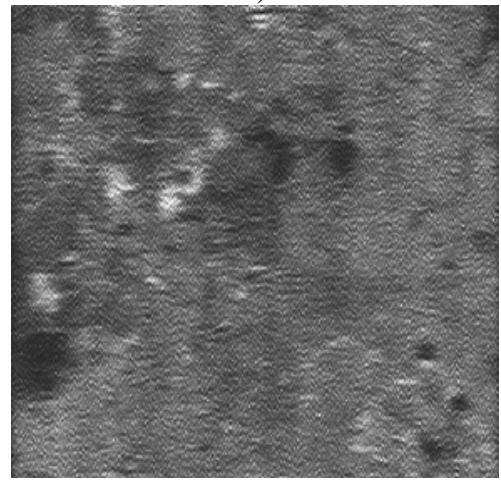

c)

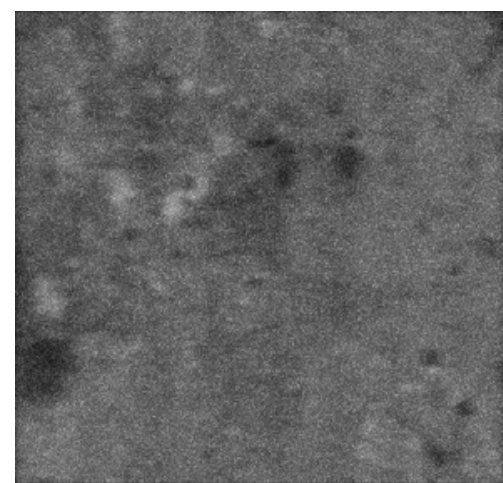

b)

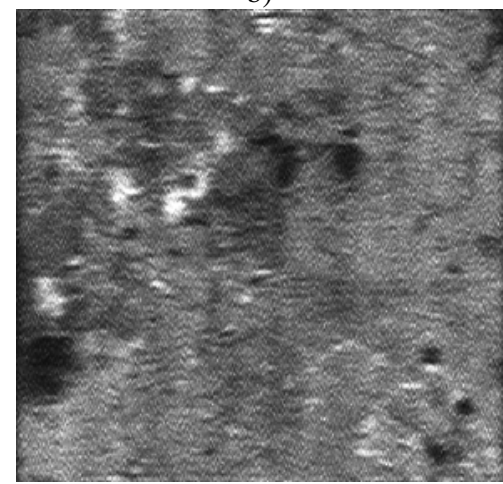

d)

Fig. 5. Operational scenario, scene $(\mu=15 \mathrm{~dB})$ : (a) original scene; (b) degraded uncertain scene image formed applying the MSF method; (c) image reconstructed applying the RSF algorithm; (d) image reconstructed applying the RASF algorithm 


\section{Simulations and Performance Analysis}

In this study, the simulations were performed with a large scale (512-by-512) pixel format image borrowed from the real-world high-resolution terrain SAR imagery (south-west Guadalajara region, Mexico [9]). The quantitative measures of the image enhancement/reconstruction performance gains achieved with the particular employed DEDR-RSF and DEDR-RASF techniques, evaluated via IOSNR metric [3],[6], are reported in Table 1 and Figure 5.

Table 1. Image enhancement of DEDR-related RSF/RASF algorithms

\begin{tabular}{|c|c|c|}
\hline $\begin{array}{c}\text { SNR } \\
{[\mathbf{d B}]}\end{array}$ & $\begin{array}{c}\text { DEDR-regularized } \\
\text { RSF Method }\end{array}$ & $\begin{array}{c}\text { DEDR-regularized } \\
\text { RASF Method }\end{array}$ \\
\hline$\mu$ & $I O S N R[\mathrm{~dB}]$ & $I O S N R[\mathrm{~dB}]$ \\
\hline 5 & 4.31 & 7.19 \\
\hline 10 & 6.15 & 9.35 \\
\hline 15 & 7.91 & 11.01 \\
\hline 20 & 9.31 & 13.12 \\
\hline
\end{tabular}

Table 2. Synthesis metrics

\begin{tabular}{|l|c|}
\hline \multicolumn{2}{|c|}{ Area of Hardware Cores } \\
\hline Logic & Utilization* \\
\hline Slice Registers, Flip Flops and Latches & $14 \%$ \\
\hline LUTs, Logic, Shift Reg. and Dual-RAMs & $25 \%$ \\
\hline BUFGs & $12 \%$ \\
\hline DSP48 & $35 \%$ \\
\hline
\end{tabular}

*The reference area is Xilinx Virtex-4 XC4VSX35-10ff668.

Next, the overall timing performances achieved with the proposed approach are reported in Table 3.

Table 3. Timing performances

\begin{tabular}{|l|c|}
\hline \multicolumn{2}{|c|}{ Timing Performance of Hardware Cores } \\
\hline Maximum Pin delay: & $9.15 \mathrm{~ns}$ \\
\hline $\begin{array}{l}\text { Average connection delay on the } \\
\text { 10 worst nets: }\end{array}$ & $9.07 \mathrm{~ns}$ \\
\hline Maximum Frequency & $109.28 \mathrm{MHz}$ \\
\hline
\end{tabular}

Last, it is compared the required processing time of two different implementation techniques as reported in Table 4. In the first case, the general-form DEDR procedure implemented in the conventional MATLAB software in a personal computer (PC) running at $1.73 \mathrm{GHz}$ with a Pentium (M) processor and $1 \mathrm{~GB}$ of RAM memory and in the second case, the same DR-related algorithms were implemented using the proposed FPGA based HW/SW co-design architecture (partitioning the Matlab application in SW and HW functions) employing the Xilinx FPGA XC4VSX35-10ff668. 
Table 4. Comparative time processing analysis

\begin{tabular}{|l|c|c|}
\hline \multicolumn{1}{|c|}{ Method } & \multicolumn{2}{|c|}{ Processing Time [secs] } \\
\hline & RSF & RASF \\
\hline $\begin{array}{l}\text { Optimized-Form DEDR } \\
\text { (PC implementation) }\end{array}$ & 19.70 & 20.05 \\
\hline Proposed HW/SW Co-design with NSA & 2.42 & 2.56 \\
\hline
\end{tabular}

\section{Concluding Remarks}

The principal result of this paper is the unified intelligent descriptive experiment design regularization (DEDR) methodology and the HW/SW Co-Design technique for enhancement/reconstruction of the remote sensing (RS) imagery using a network of Systolic Arrays (NSA) in a computationally efficient fashion that meets the (near) real time imaging system requirements. We do believe that pursuing the addressed HW/SW co-design paradigm based on NSAs, one could definitely approach the real time image processing requirements while performing the reconstruction of the large-scale real-world RS imagery. Finally, the processing time of the DEDR RSF/RASF algorithms were significantly reduced up to eight times compared with the computational time of the Optimized-form DEDR procedure implemented in the conventional MATLAB software.

\section{References}

1. De Micheli, G., Gupta, R.K.: Hardware-Software Codesign. Proceedings of the IEEE V85(3) (1997)

2. Greco, J., Cieslewski, G., Jacobs, A., Troxel, I.A., George, A.D.: Hardware/software interface for high-performance space computing with FPGA coprocessors. In: Aerospace Conference, March 4-11, pp. 10-25. IEEE, Los Alamitos (2006)

3. Shkvarko, Y.V.: Unifying Regularization and Bayesian Estimation Methods for Enhanced Imaging with Remotely Sensed Data. Part I and Part II. In: IEEE Transactions on Geoscience and Remote Sensing, vol. 42, pp. 923-931. IEEE, Los Alamitos (2004)

4. Shkvarko, Y.V., Perez Meana, H.M., Castillo Atoche, A.: Enhanced radar imaging in uncertain environment: A descriptive experiment design regularization paradigm -Accepted for publication. Intern. Journal of Navigation and Observation (2008) (in press)

5. Ponomaryov, V.I.: Real-time 2D-3D filtering using order statistics based algorithms. Journal Real-Time Image Processing 1, 173-194 (2007)

6. Castillo Atoche, A., Shkvarko, Y.V., Perez Meana, H.M., Torres Roman, D.: Convex Regularization-Based Hardware/Software Co-Design for Real-Time Enhancement of Remote Sensing Imagery. Int. Journal of Real Time Image Processing (in press)

7. Meyer Baese, U.: Digital Signal Processing with Field Programmable Gate Array. Springer, Berlín (2001)

8. EDK 9.1 MicroBlaze tutorial in Virtex-4, Xilinx, http: / / www . xilinx. com

9. Space imaging in GeoEye Inc. (2008), http: / / www. spaceimaging.com/quicklook

10. Lo, S.C., Jean, S.N.: Mapping Algorithms to VLSI Array Processors. In: International Conference on Acoustics, Speech, and Signal Processing (1988)

11. Kung, S.Y.: VLSI Array Processors. Prentice Hall, Englewood Cliffs (1988) 